\title{
Diagnostic accuracy of ultrasonography in the assessment of anterior knee pain
}

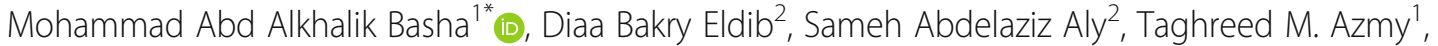 \\ Nader E. M. Mahmoud', Tarek Mohamed Ghandour ${ }^{3}$, Tarek Aly ${ }^{4}$, Shimaa Mostafa ${ }^{5}$, Asmaa M. Elaidy ${ }^{6}$ and \\ Hesham Youssef Algazzar ${ }^{2}$
}

\begin{abstract}
Background: Anterior knee pain (AKP) is a problematic complaint, considered to be the most frequent cause of orthopedic consultancy for knee problems. This study aimed to highlight diagnostic accuracy of ultrasonography as a fast imaging technique in assessment of patients with AKP.

Methods and results: A prospective study was conducted on 143 patients with clinically confirmed AKP. All patients underwent ultrasonography and MRI examinations of the knee. The diagnostic accuracy of ultrasonography compared to MRI for evaluating different findings of possible causes of AKP were analyzed using receiver operating characteristic (ROC) curve and judged by area under curve (AUC). A total of 155 knees were included in the study; 26 knees showed no abnormalities, 19 knees showed positive MRI only, and 110 knees showed positive ultrasonography and MRI. Ultrasonography and MRI reported 11 different findings of possible causes of AKP or related to it. Joint effusion was the most common finding (38\%) followed by trochlear cartilage defect (20.6\%) and superficial infrapatellar subcutaneous edema (20\%). The overall accuracy of ultrasonography was $85.3 \%$ sensitivity and $100 \%$ specificity. The ultrasonography provided the highest sensitivity (100\%) in detecting bipartite patella, followed by $91.5 \%$ for joint effusion, and $87.5 \%$ for quadriceps tendinopathy. The ROC curve analysis of overall accuracy of ultrasonography showed an AUC of 0.93. The overall Kappa agreement between ultrasonography and MRI was good $(k=0.66)$.
\end{abstract}

Conclusion: Ultrasonography can be used to make a swift screening and assessment of painful anterior knee and as an alternative to MRI when it is unavailable or contraindicated.

Keywords: Knee joint, Ultrasonography, Magnetic resonance imaging, Anterior knee pain, Diagnostic accuracy

\section{Key points}

- Ultrasonography showed high diagnostic accuracy in detecting most causes of AKP.

- Although MRI is the gold standard technique for AKP imaging, ultrasonography can be used to make

\footnotetext{
* Correspondence: Mohammad_basha76@yahoo.com

'Department of Radiodiagnosis, Faculty of Human Medicine, Zagazig

University, Zagazig, Egypt

Full list of author information is available at the end of the article
}

a swift screening and assessment of the painful anterior knee and can be used as an alternative to MRI when MRI is unavailable or contraindicated.

- MRI may be warranted if a patellar cartilage defect is clinically suspected or the ultrasonography yielded negative results.

\section{Background}

Anterior knee pain (AKP) is a problematic complaint, considered to be the most frequent cause of

\section{Springer Open}

(c) The Author(s). 2020 Open Access This article is licensed under a Creative Commons Attribution 4.0 International License, which permits use, sharing, adaptation, distribution and reproduction in any medium or format, as long as you give appropriate credit to the original author(s) and the source, provide a link to the Creative Commons licence, and indicate if changes were made. The images or other third party material in this article are included in the article's Creative Commons licence, unless indicated otherwise in a credit line to the material. If material is not included in the article's Creative Commons licence and your intended use is not permitted by statutory regulation or exceeds the permitted use, you will need to obtain permission directly from the copyright holder. To view a copy of this licence, visit http://creativecommons.org/licenses/by/4.0/. 
orthopedic consultancy for knee problems [1]. AKP's differential diagnosis falls within a wide range, which is still overlapping and lacking clear understanding [2]. It is more frequent in young people between 15 and 30 years and is more frequent in women than in men. Despite its prevalence, AKP nature and causes remain inadequately understood and can be bothersome for patients and clinicians as it causes chronic disability, limited sports and activity, and a negative impact on the quality of life [3, 4]. Many authors linked AKP to the patellofemoral pathology, especially the patellofemoral instability, while others clearly reported that structural anomalies did not provide a complete explanation of the pain [5]. Moreover, structural anomalies were found to be minor among AKP patients, and there was no obvious correlation between patellofemoral malalignment and long-term results of AKP treatment [6].

The patient's clinical history and physical examination are of paramount importance in diagnosing the cause of AKP. The physical examination is complemented by imaging examinations, and the combination should yield a precise diagnosis that will be the cornerstone in developing an appropriate therapeutic program [7]. Imaging workup is important to evaluate the extent of the bone and soft tissue abnormality and guide therapeutic intervention if needed [8,9]. The most helpful diagnostic techniques for evaluating soft tissue changes are ultrasonography and magnetic resonance imaging (MRI). Plain radiography is of limited value, and computed tomography is not recommended [10, 11]. Most orthopedics rely on MRI as the method of choice in knee imaging as it provides high contrast resolution images not only for the soft tissue but also for the underlying bone and allowing a precise assessment of the underlying cause [3, 12]. Moreover, MRI has replaced diagnostic arthroscopy as the primary diagnostic modality for many knee pathologies [13]. Ultrasonography has become more popular because it is safe, quick, inexpensive, and reliable. It has the ability to assess soft tissues in the anterior aspect of the knee, which could be the main source of pain [6].

Although the literature is full of several researches about the value of ultrasonography examination of the knee, the AKP dilemma makes the need for evidence-based value, regarding various diagnoses, useful for guiding choices in value-based health care in imaging, a point that should be stressed. Therefore, we conducted our prospective study to clarify the diagnostic accuracy of ultrasonography as a fast imaging technique in the assessment of patients with AKP and comparing the results with MRI.

\section{Methods}

\section{Ethical statement}

Ethical approval was obtained from the local research ethical committee. All patients were informed of the study and provided written informed consent prior to ultrasound and MRI examination. The study was performed in accordance with the ethical principles of the Declaration of Helsinki.

\section{Study population}

This prospective study was carried out between August 2018 and January 2020. Inclusion criteria were patients with a clinically confirmed AKP and scheduled for MRI examination of the knee. Initially, we collected 200 consecutive patients. Exclusion criteria were (i) patients with a history of patellofemoral malalignment since the ultrasonography diagnostic criteria for patellofemoral malalignment is not established yet $(n=19)$, (ii) patients who underwent surgeries or previously fractured knee joint $(n=34)$, and (iii) patients who had absolute contraindications to MRI examination $(n=4)$. The exclusion process resulted in a final cohort comprised of 143 patients. Fig. 1 illustrates the flow chart of our study.

\section{Ultrasonography and color Doppler technique and analysis}

Gray-scale and color Doppler ultrasonography of the knee were performed using Aplio 400, Toshiba ultrasound scanner, with a high-resolution, multifrequency linear transducer $(7-12 \mathrm{MHz})$. Patients were positioned in a supine position with the knee comfortably flexed $\left(30-45^{\circ}\right)$ by placing a pillow under the knee. To avoid loss of contact, we used plenty of thick gel. The exam started at the suprapatellar region by scanning in the long axis plane from medial to lateral. The quadriceps tendon was scanned first in both long axis and short axis planes. The trochlear cartilage, as well as the medial and lateral patellar recesses, was examined in various degrees of knee flexions. Long and short axis planes for the patellar tendon were then obtained. The parameters of the color Doppler mode was set to depict the slow flows by using high Doppler frequency, low pulse repetition frequency, minimal wall filter, and high color gain. Focus is positioned just deep to the area of interest. Any suspected lesion, firmness, or tenderness was examined by moving the probe over and around the lesion [14-16]. Dynamic ultrasonography examination executed by changing the degree of knee flexion as well as by medial and lateral movements of the patella was also done. During the ultrasonography examination, the knee was divided into the following entities: (i) the extensor mechanism, i.e., quadriceps tendon, patella, and patellar tendon; (ii) the trochlear femoral articular cartilage; (iii) anterior knee joint recesses (suprapatellar and both 


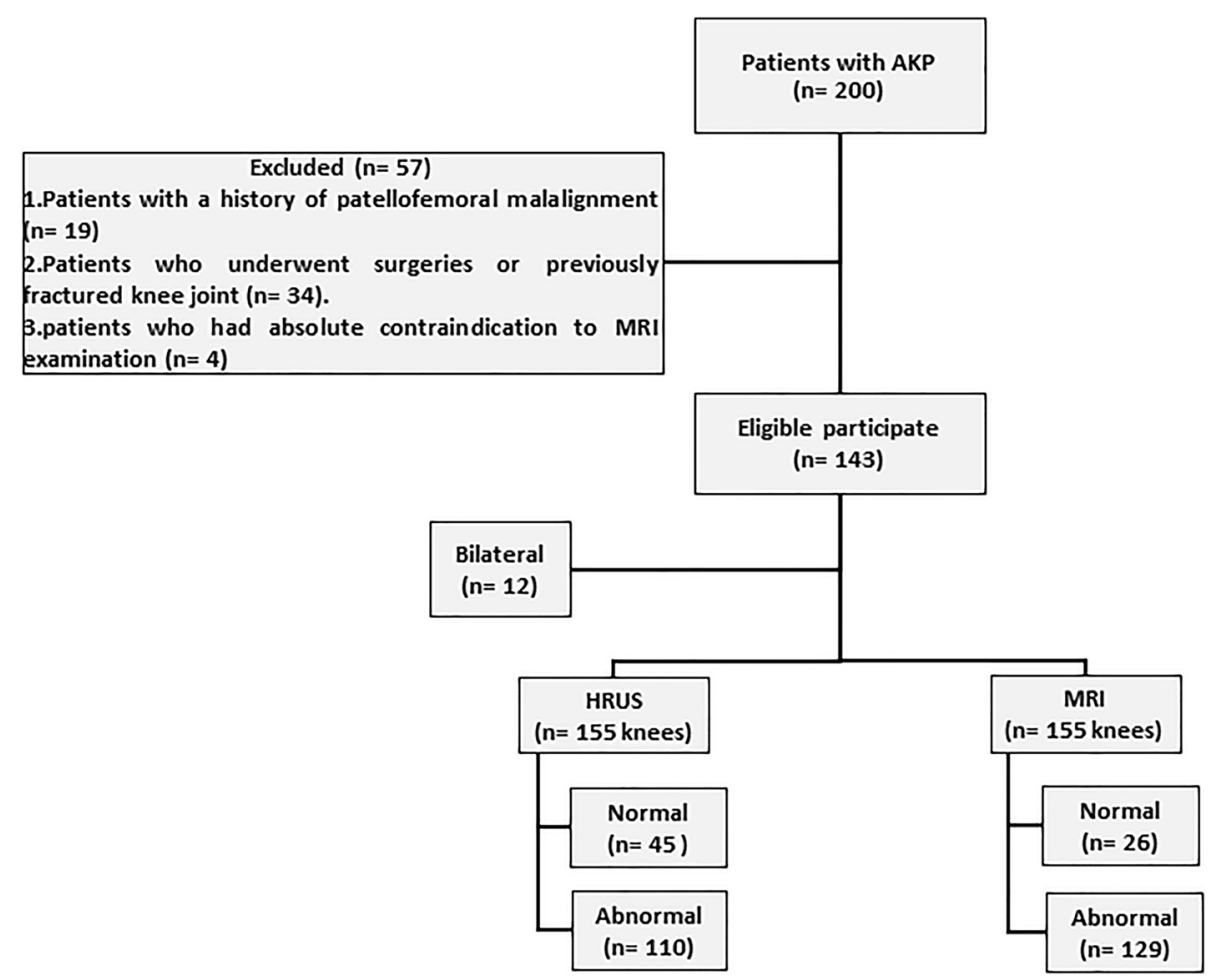

Fig. 1 Flow chart of our study. AKP, anterior knee pain; n, number; MRI, magnetic resonance imaging

medial and lateral recesses); (iv) anterior knee bursa, i.e., subcutaneous prepatellar bursa, subcutaneous infrapatellar bursa, and deep infrapatellar bursa; (v) suprapatellar fat and deep infrapatellar Hoffa's fat pad; and (vi) miscellaneous causes.

All ultrasonography examinations were performed by one highly experienced musculoskeletal radiologist (with over 10 years of musculoskeletal ultrasound experience and had performed $>1000$ ultrasound examinations per year). The radiologist was blinded to clinical data. Ultrasonography findings of AKP are summarized in Fig. 2. Hypervascularity on color Doppler examinations was used as a marker for patellar tendinopathy, Hoffa's or suprapatellar fat pad impingement, and inflammation.

\section{MRI protocol and image analysis}

MRI was performed during a timeframe of 1 week after the ultrasonography. The applied MRI protocol was revised to ensure its compliance with the study requirements. MRI was performed using 1.5-T Toshiba Vantage Elan System. A Flex Speeder 16 channel (receiver only) coil was used. The MRI protocol consisted of fat-suppressed and non-fatsuppressed sequences. The protocol for the evaluation of the knee is listed in Table 1 .
All MRI data were transferred to the workstations, and image analysis was performed on the PACS system (PaxeraUltima-paxeramed). All MRI images were interpreted by one radiologist with over 17 years of experience in musculoskeletal imaging. The radiologist was blinded to the clinical history and ultrasound findings. MRI findings of AKP are summarized in Fig. 2.

\section{Statistical analysis}

Demographic data are presented as frequency and percentages. Diagnostic accuracy of ultrasonography included sensitivity, specificity, positive predictive values (PPV), and negative predictive value (NPV) as compared to MRI for evaluating different anterior knee findings were analyzed using receiver operating characteristic curve (ROC) and judged by the area under the curve (AUC). The diagnostic significance of the determined AUC was compared to the null hypothesis that $\mathrm{AUC}=0.5$, which is the area under the diagonal reference line. We use the method of Delong et al. [17] for the calculation of the standard error of the AUC. Cohen's $\kappa$ was run to determine if there was an agreement between the ultrasonography and MRI on identifying different lesions in patients' knees. Interpretation of $\kappa$-statistic value is based on Altman [18] as moderate agreement $(\kappa=0.41-0.6)$, good 

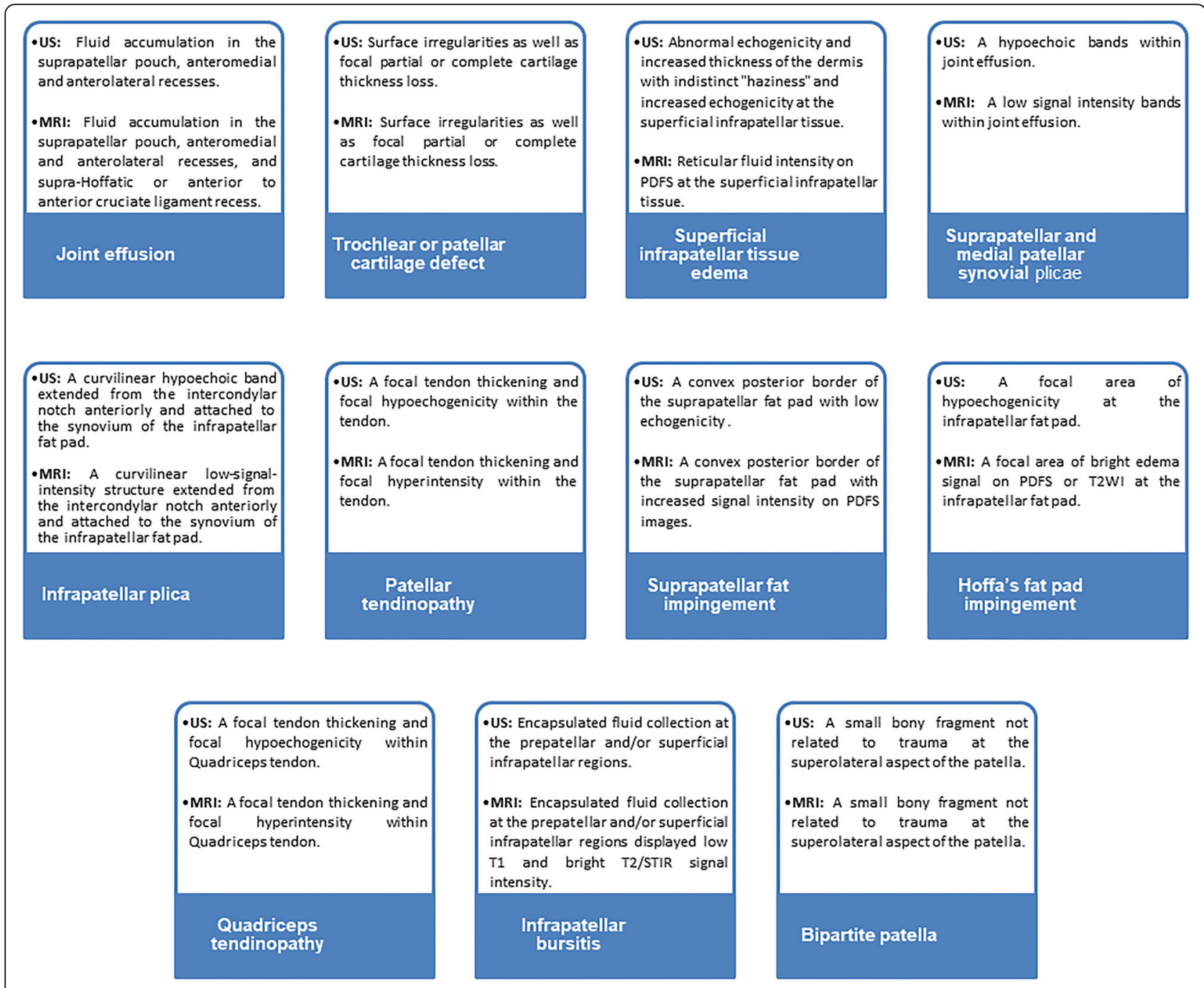

Fig. 2 Ultrasonography and MRI findings of AKP. US, ultrasonography; MRI, magnetic resonance imaging; AKP, anterior knee pain; PDFS, proton density fat sat; STIR, short tau inversion recovery

agreement $(\kappa=0.61-0.80)$, and very good agreement $(\kappa=0.81-1)$. Statistical analysis was conducted using Medcalc software version 19.1, 2019, and IBM SPSS statistics version 26, 2019, for Windows statistical package. A $p$ value $\leq 0.05$ is considered statistically significant.

\section{Results}

\section{Patients}

A total of 155 knees from 143 patients (80 males and 63 females; mean age, $33.6 \pm 13.9$ years; range, $12-62$ years) were included in our study. The most common age group was between 26 and 35-year-old (30\%). Twelve

Table $1 \mathrm{MRI}$ protocol for evaluation of the knee

\begin{tabular}{|c|c|c|c|c|c|c|c|}
\hline & TR (ms) & TE (ms) & Thickness (mm) & Interslice gap (\%) & FOV $(\mathrm{cm})$ & Matrix & Scan time $(\min )$ \\
\hline T2 FSE axial & $3200-3800$ & 110 & 3 & 10 & $17 \times 17$ & $356 \times 286$ & 3.25 \\
\hline PDFS axial & $2100-2600$ & 36 & 3 & 10 & $16 \times 16$ & $284 \times 256$ & 3.40 \\
\hline PDFS sagittal & $2100-2600$ & 36 & 3 & 10 & $16 \times 16$ & $284 \times 256$ & 3.04 \\
\hline T1 FSE sagittal & $400-410$ & 10 & 3 & 10 & $16 \times 16$ & $56 \times 286$ & 2.5 \\
\hline PDFS coronal & $2100-2600$ & 36 & 3 & 10 & $16 \times 16$ & $284 \times 256$ & 2.45 \\
\hline
\end{tabular}

FSE fast spine echo, PDFS proton density fat sat, TR repetition time, TE echo time, FOV field of view, MRI magnetic resonance imaging, ms millisecond, $\mathrm{cm}$ centimeter, min minutes 
Table 2 Number of findings in each knee as detected by ultrasonography and MRI

\begin{tabular}{lll}
\hline Number of findings & Ultrasonography & MRI \\
\hline No findings & 45 & 26 \\
One finding & 49 & 52 \\
Two findings & 42 & 44 \\
Three findings & 14 & 17 \\
Four findings & 5 & 12 \\
Five findings & 0 & 4
\end{tabular}

The data represent the number of affected knees $M R I$ magnetic resonance imaging

patients $(8.4 \%)$ had bilateral AKP. The chief complaint in our patients was knee pain or disability, not more than 6 months.

\section{Ultrasonography and MRI findings}

The ultrasonography detected 195 findings in 110 knees, compared to 259 findings in 129 knees detected by MRI. Ultrasonography revealed 45 knees with no findings compared to 26 knees by MRI. The number of findings per technique is shown in Table 2 . Both ultrasonography and MRI reported 11 different findings of possible causes of AKP or related to it (Table 3). Joint effusion was the most common finding (59 patients, 38.1\%), while trochlear cartilage defect and superficial infrapatellar subcutaneous edema were reported in $20.6 \%$ and $20 \%$, respectively (Fig. 3). Ultrasonography was not able to detect any of 23 knees with patellar cartilage defect and detected 23 out of 32 knees with trochlear cartilage defects. MRI

Table 3 Frequency of each finding as detected by ultrasonography

\begin{tabular}{lllll}
\hline & TP & FP & FN & TN \\
\hline Joint effusion & 54 & 4 & 5 & 92 \\
Trochlear cartilage defect & 23 & 2 & 9 & 121 \\
Superficial infrapatellar tissue edema & 24 & 0 & 7 & 124 \\
Synovial plica & 22 & 0 & 6 & 127 \\
Patellar tendinopathy & 21 & 0 & 4 & 130 \\
Patellar cartilage defect & 0 & 0 & 23 & 132 \\
Suprapatellar fat impingement & 16 & 0 & 3 & 136 \\
Hoffa's fat pad impingement & 12 & 0 & 6 & 137 \\
Quadriceps tendinopathy & 14 & 0 & 2 & 139 \\
Infrapatellar bursitis & 4 & 0 & 2 & 149 \\
Bipartite patella & 2 & 0 & 0 & 153 \\
\hline TR true positive, FP false positive, $F N$ false negative, $T N$ true negative &
\end{tabular}

$T R$ true positive, $F P$ false positive, $F N$ false negative, $T N$ true negative showed 28 knees with evidence of plica: 13 suprapatellar plicae, 10 medial plicae, and 5 infrapatellar plicae. Ultrasonography detected 12 out of the 13 suprapatellar plicae and all medial patellar plicae. Ultrasonography was not able to detect the infrapatellar plicae. Ultrasonography detected 21 out of 25 knees with patellar tendinopathy, 14 out of 16 knees with quadriceps tendinopathy, and 16 out of 19 knees with suprapatellar pad of fat impingement. MRI showed 18 knees with Hoffa's fat pad impingement; 12 were identified by ultrasonography, and 6 were missed. Three out of 12 knees showed increase vascularity on color Doppler examination. Ultrasonography detected the two knees with bipartite patella and 4 out of 6 knees with infrapatellar bursitis.

\section{Diagnostic accuracy of ultrasonography}

Out of the 155 knees, 26 (16.8\%) showed no abnormalities by both ultrasonography and MRI, 110 (71\%) showed positive findings by both ultrasonography and MRI, and 19 (12.2\%) showed positive findings by MRI, whereas ultrasonography was reported to be normal. The overall diagnostic accuracy of ultrasonography in detecting the abnormal findings in AKP patients was $85.3 \%$ sensitivity, $100 \%$ specificity, $100 \%$ PPV, and $57.8 \%$ NPP. The ultrasonography provided the highest sensitivity $(100 \%)$ in detecting the bipartite patella, followed by $91.5 \%$ for joint effusion, $87.5 \%$ for quadriceps tendinopathy, $84.2 \%$ for suprapatellar fat impingement, and $84 \%$ for patellar tendinopathy (Table 4).

\section{ROC curve analysis}

Table 4 summarizes the ROC curve and the Cohen Kappa analysis for each finding. The ROC curve analysis of the diagnostic accuracy of ultrasonography for detecting the abnormal findings in AKP patients in comparison to MRI showed that the highest diagnostic accuracy was in detecting quadriceps tendinopathy (AUC $=0.94, p<0.001)$, followed by joint effusion (AUC $=0.93, p<0.001)$, and impingement of suprapatellar fat $(\mathrm{AUC}=0.92, p<0.001)$. The ROC curve analysis of the overall diagnostic accuracy of ultrasonography showed an AUC of 0.93 (95\% CI $=0.72-$ 0.91, $p<0.001$ ) (Fig. 4). The overall Kappa agreement between ultrasonography and MRI was good $(k=$ 0.66, 95\% CI $=0.53-0.80$ ).

Representative cases of our study are shown in Figs. 5, 6, 7, 8, 9, and 10 .

\section{Discussion}

The current study highlighted the role of ultrasonography in the diagnosis of AKP. The overall results are encouraging and demonstrated the high diagnostic 


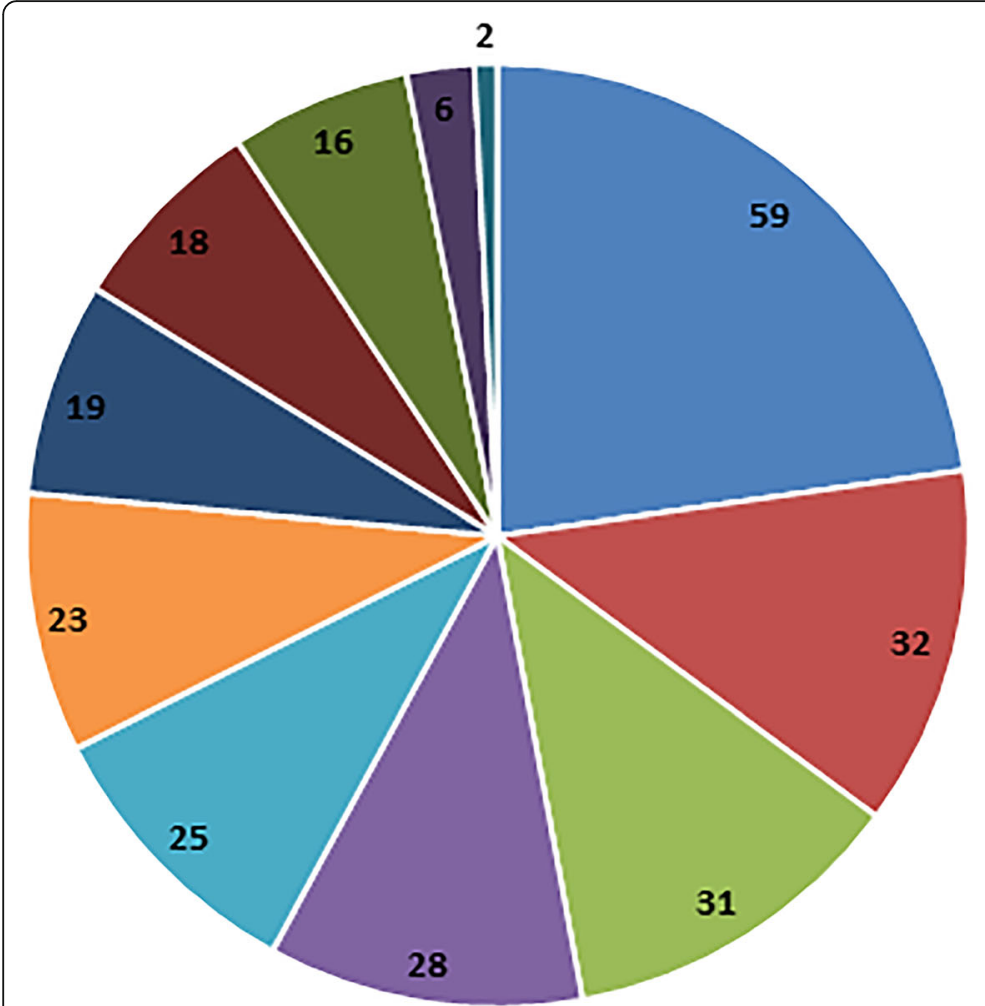

a Joint effusion

- Trochlear cartilage defect

* Superficial infrapatellar tissue edema

a Synovial plica

a Patellar tendinopathy

- Patellar cartilage defect

- Sup rapatellar fat impingement

- Hoffa's fat pad impingement

- Quadriceps tendinopathy

- Infrapatellar bursitis

- Bipartite patella

Fig. 3 Frequency of AKP findings as detected by MRI. AKP, anterior knee pain; MRI, magnetic resonance imaging

Table 4 Diagnostic accuracy of ultrasonography findings using MRI as the gold reference standard

\begin{tabular}{|c|c|c|c|c|c|c|}
\hline & Sensitivity (\%) & Specificity (\%) & PPV & NPV & AUC (ROC) & $\begin{array}{l}\text { Kappa agreement } \\
\text { (Cohen's Kappa) }\end{array}$ \\
\hline Joint effusion & 91.5 & 95.8 & 93.1 & 94.8 & $0.93^{* * *}$ & $0.87^{* * *}$ \\
\hline Trochlear cartilage defect & 71.9 & 98.4 & 92 & 93.1 & $0.85^{* * *}$ & $0.76^{* * *}$ \\
\hline Superficial infrapatellar tissue edema & 77.4 & 100 & 100 & 94.7 & $0.88^{* * *}$ & $0.83^{* * *}$ \\
\hline Synovial plica & 78.6 & 100 & 100 & 95.5 & $0.88^{* * *}$ & $0.84^{* * *}$ \\
\hline Patellar tendinopathy & 84 & 100 & 100 & 97 & $0.92^{* * *}$ & $0.89^{* * *}$ \\
\hline Patellar cartilage defect & 0 & 100 & - & 85.2 & $0.5^{\#}$ & $0^{*}$ \\
\hline Suprapatellar fat impingement & 84.2 & 100 & 100 & 97.8 & $0.92^{* * *}$ & $0.9^{* * *}$ \\
\hline Hoffa's fat pad impingement & 66.7 & 100 & 100 & 95.8 & $0.83^{* * *}$ & $0.775^{* * *}$ \\
\hline Quadriceps tendinopathy & 87.5 & 100 & 100 & 98.6 & $0.94^{* * *}$ & $0.93^{* * *}$ \\
\hline Infrapatellar bursitis & 66.7 & 100 & 100 & 98.4 & $0.83^{¥ *}$ & $0.79^{* * *}$ \\
\hline Bipartite patella & 100 & 100 & 100 & 100 & $1^{* * *}$ & $1^{* * *}$ \\
\hline Overall validity & 85.3 & 100 & 100 & 57.8 & $0.93^{* * *}$ & $0.66^{* * *}$ \\
\hline
\end{tabular}

$\overline{M R I}$ magnetic resonance imaging, $P P V$ positive predictive value, NPV negative predictive value, $C I$ confidence interval, $A U C$ area under curve, $R O C$ receiver operating characteristic curve

${ }_{* * *} p$ value $<0.001$

\# $p$ value $>0.05$ (non-significant)

${ }^{*}$ No $p$ value can be calculated

$¥ ¥ p$ value $=0.002$ 


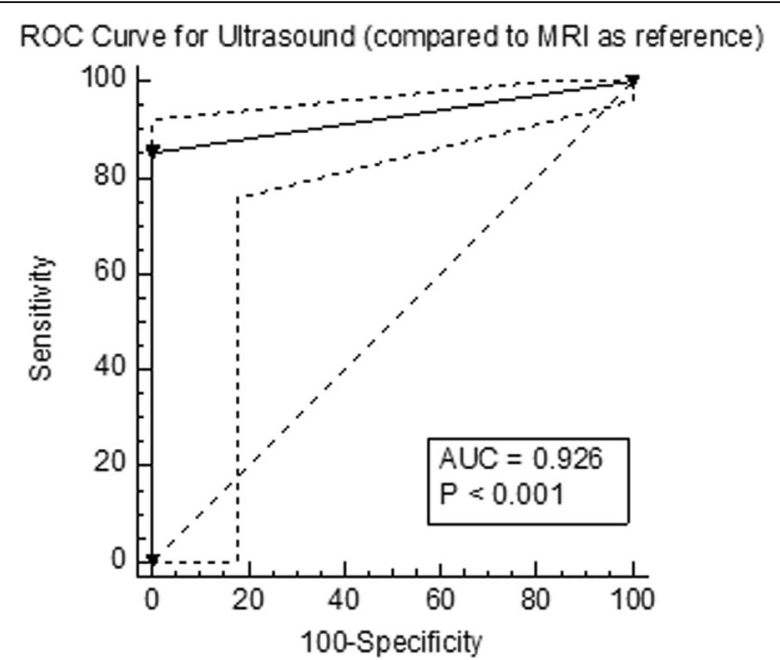

Fig. 4 ROC curve analysis of ultrasonography for the identification of anterior knee findings compared to MRI. ROC, receiver operating characteristic curve; MRI, magnetic resonance imaging; AUC, area under the curve

accuracy of ultrasonography $(85.3 \%$ sensitivity, $100 \%$ specificity, $100 \% \mathrm{PPV}$, and $57.4 \% \mathrm{NPV}$ ) compared to MRI. Ultrasonography detected 195 out of 259 findings detected by MRI. Some of these findings could be the cause of the AKP like tendinopathy, fat impingement, and chondropathy, whereas others were just a sign of associated pathology like joint effusion and anterior subcutaneous edema, which may strengthen an equivocal or suspected clinical diagnosis. The ultrasonography detected most of the pathological findings in this study. The early and fast diagnosis by ultrasonography could allow the orthopedic to start the patient management plane without the usual delay for requesting and scheduling MRI examinations that would have a good impact on patient recovery.

According to the study done by Artul et al. [19], 34\% of ultrasonography reports were negative, and $66 \%$ were positive. In the present study, 29\% (45 knees) of ultrasonography reports and $16.8 \%$ (26 knees) of MRI reports were normal.

Lee and Chow [20] reported that ultrasonography is a sensitive tool to assess knee joint effusion, and a minimal amount of joint effusion as low as 7 to $10 \mathrm{ml}$ could be optimally detected. According to the Draghi et al. [21] study, ultrasonography had $81.3 \%$ sensitivity and $100 \%$ specificity in detecting knee effusion. In the present study, ultrasonography showed a sensitivity of $91.5 \%$ and specificity of $95.8 \%$ in detecting knee effusion. Ultrasonography failed to detect five knees as the effusions were minimal and presented in front of the anterior cruciate ligament. On the other hand, possible
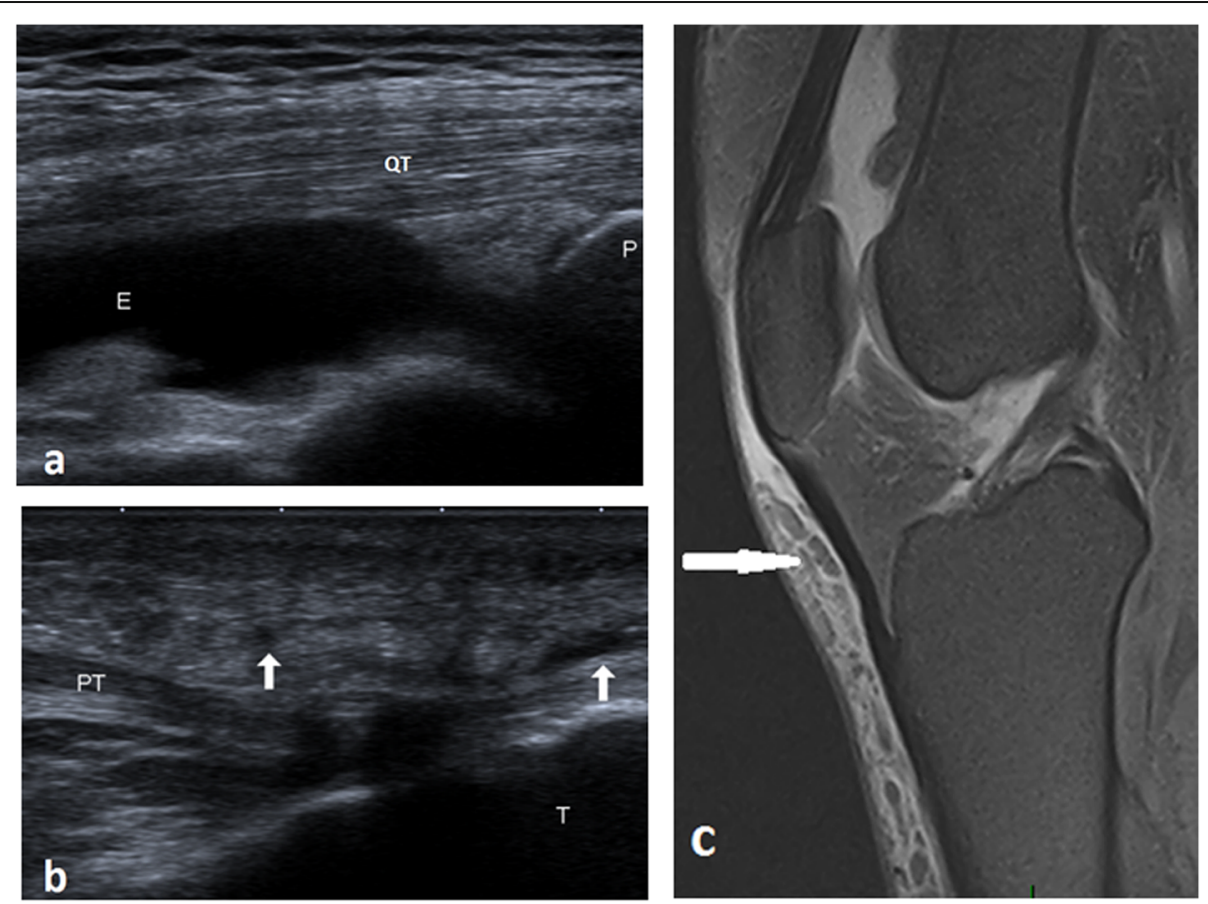

Fig. 5 A 44-year-old male with joint edema and anterior subcutaneous edema. a Long axis ultrasound image illustrates joint effusion in the suprapatellar pouch. b Long axis ultrasound scan illustrates hypoechoic subcutaneous reticulations anterior to the patellar tendon (arrows). $\mathbf{c}$ Sagittal PDFS MRI illustrates joint effusion, prepatellar, and superficial infrapatellar reticular fluid intensities of edema. QT, quadriceps tendon; PT, patellar tendon; E, effusion; P, patella; T, tibia 

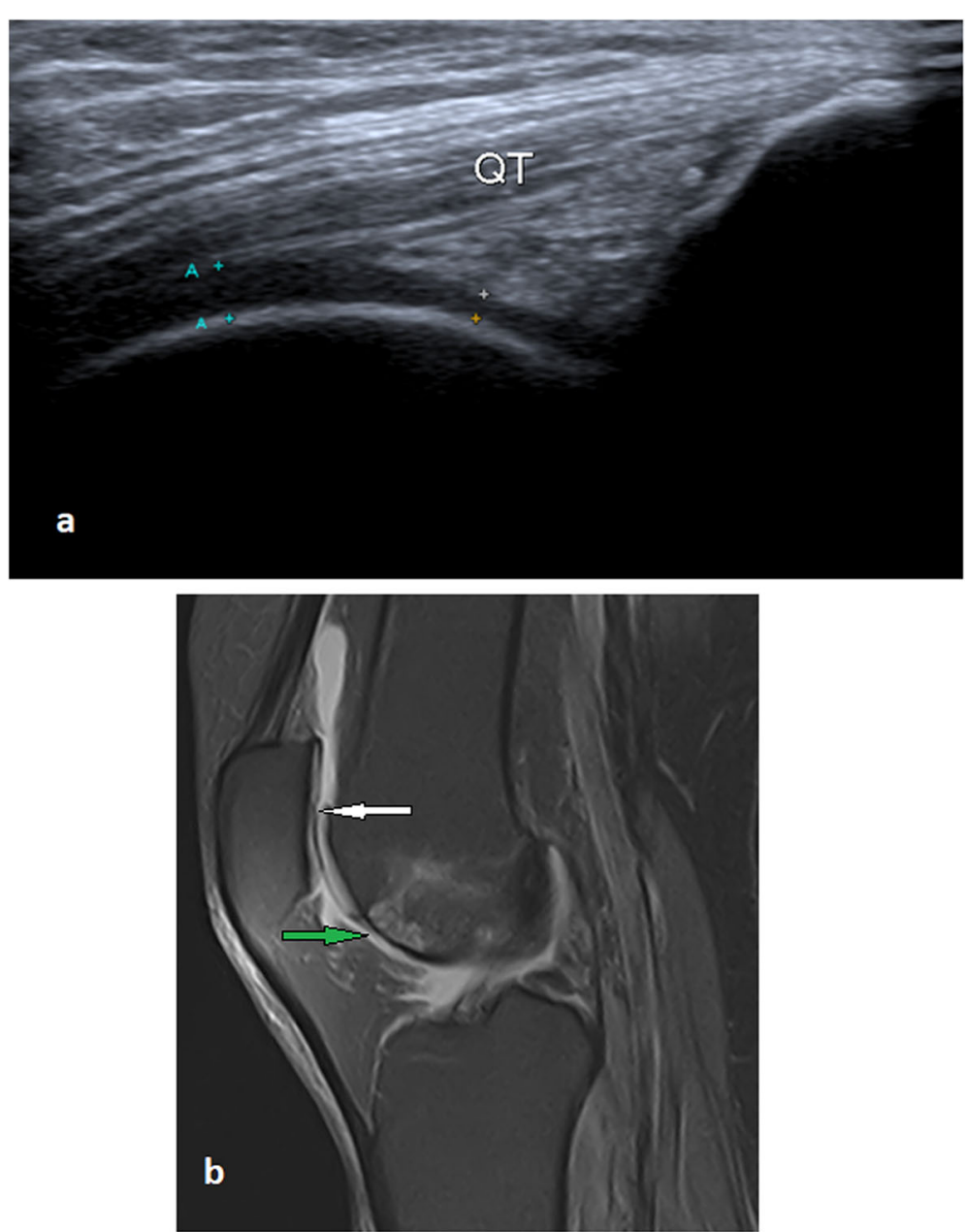

Fig. 6 A 54-year-old male with focal patellar and trochlear partial thickness cartilage loss, mild joint effusion, and anterior infrapatellar subcutaneous edema of the left knee. a Long axis ultrasound image of the left knee illustrates a focal thinning of the anteroinferior aspect of the trochlear cartilage. b Sagittal PDFS MRI illustrates partial thickness cartilage loss of the patella (white arrow), partial-thickness cartilage loss of the anteroinferior surface of the trochlear cartilage with subchondral bone marrow changes (green arrow), joint effusion, and anterior subcutaneous edema. QT, quadriceps tendon

improvement in the gap period between ultrasonography and MRI may be responsible for the false-positive results.

In the relevant literature, the detection of cartilage defects commonly refers to trochlear cartilage. The patellar cartilage is not routinely assessed by ultrasonography, as it is usually shaded by the patella. Cao et al. [22] stated that ultrasonography is considered a promising screening tool for assessment of trochlear cartilage defects as it showed a sensitivity range of 62.2 to $69.4 \%$ and specificity range of 90.5 to $92.9 \%$. In the present study, ultrasonography showed a sensitivity of $71.9 \%$ and a specificity of $98.4 \%$ in detecting trochlear cartilage defects. In the 9 false-negative knees, the trochlear defects were overlooked by ultrasonography as they were deeply located in the intercondylar fossa. All patellar cartilage defects were overlooked by ultrasonography because they were obscured by the patellar shadow during the examination.

Unlu et al. [23] reported that anterior subcutaneous edema is a common finding $(82.7 \%)$ on routine knee MRI and is significantly associated with old age, overweight, and patellofemoral chondral changes. In the present study, ultrasonography showed a sensitivity of $77.4 \%$ in detecting subcutaneous edema.

Knee's synovial plica syndrome is a commonly overlooked cause of AKP [24]. Anatomically, the infrapatellar plica is the most common plica, followed by suprapatellar plica, and lastly, the medial patellar 

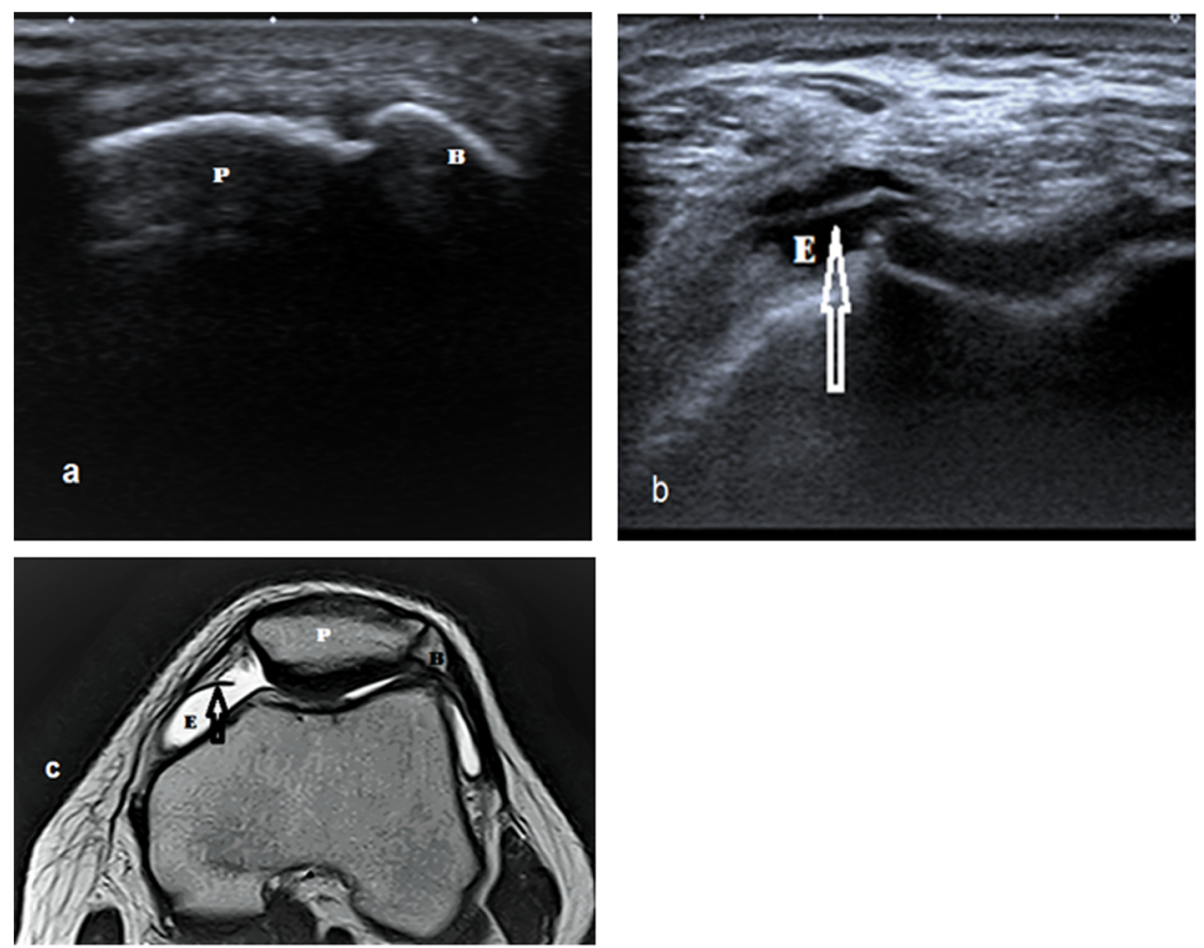

Fig. 7 A 31-year-old male with a bipartite patella, joint effusion, and medial patellar plica. a Short axis ultrasound image illustrates the cleft between the patella and bipartite fragment at the superolateral pole of the patella. $\mathbf{b}$ Short axis ultrasound image illustrates joint effusion and medial patellar plica (arrow). c Axial T2WI MRI illustrates bipartite patella, joint effusion, and medial patellar plica. P, patella; E, effusion; B, bipartite patellar fragment

plica, which is considered the most symptomatic [25]. In this study, ultrasonography showed a sensitivity of $78.5 \%$ and specificity of $100 \%$ in detecting synovial plicae. The infrapatellar plica was the least common type of plica in our study, contrary to what was reported by Kheiralla [25], probably due to the type of our group, as they were symptomatic for AKP.

Patellar tendinopathy is a common cause of AKP, especially in athletes. It mainly affects the proximal aspect of the tendon. Ultrasonography had $87 \%$ sensitivity in detecting patellar tendinopathy $[26,27]$. In this study, the sensitivity of ultrasonography was $84 \%$.

To the best of our knowledge, detection of quadriceps tendon pathology has been reported while commonly referring to the quadriceps tendon tear in athletes, and most of the related literature focused only on MRI $[25,28]$. In this study, ultrasonography showed $87.5 \%$ sensitivity, compared to $72.5 \%$ reported by King et al. [29].

Fat pad impingement syndromes most often affect the suprapatellar and superolateral Hoffa's fat pads $[30,31]$. These fat pads normally act to promote both joint lubrication and joint stability [32]. When impinged due to patellar maltracking, these fat pads display obscuration of normal fat and increased vascularity in these regions [33]. Gutierrez et al. [34] ensured the ability of ultrasonography in the detection of suprapatellar impingement. Tsavalas and Karantanas [35] reported a prevalence rate of $12 \%$ among patients with AKP. In our results, the prevalence rate of the suprapatellar fat impingement was $12.3 \%$. Ultrasonography had $84.2 \%$ sensitivity and $100 \%$ specificity in detecting suprapatellar fat impingement. Color Doppler ultrasound showed increased vascularity in 3 knees due to concomitant inflammation.

Draghi et al. [21] reported that MRI is the modality of choice in the assessment of pathological changes of the Hoffa's fat pad. Mikkilineni et al. [36] prospectively emphasized that ultrasonography may be valuable for the diagnosis of impingement of the Hoffa's fat pad, which needs more research. In this study, the sensitivity and specificity of ultrasonography were $66.7 \%$ and $100 \%$, respectively for diagnosing impingement of the Hoffa's fat pad. To our knowledge, no results were published in the literature to compare.

According to Draghi et al. [37], ultrasonography had $100 \%$ sensitivity and specificity in detecting deep 

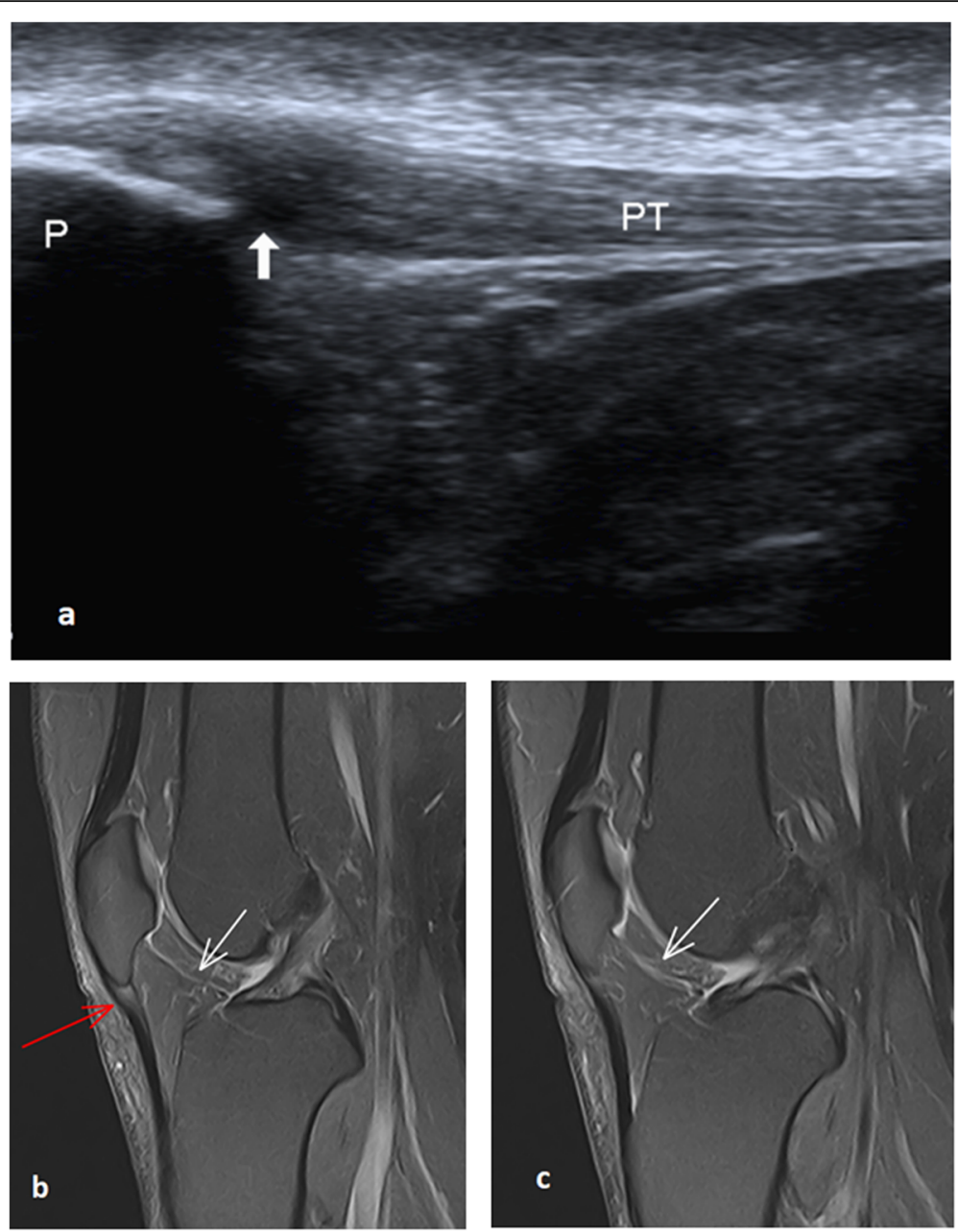

Fig. 8 A 45-year-old female with right patellar tendinopathy and infrapatellar plica. a Long axis ultrasound image through the patellar tendon shows a focal thickening of the proximal part of the patellar tendon with a localized hypoechoic area sparing the anterior fibers. (White thick arrow). $\mathbf{b}$ and $\mathbf{c}$ Two subsequent sagittal PDFS MRI images through intercondylar notch show focal proximal tendon thickening and increased signal intensity (red arrow) sparing the anterior tendon fibers. Infrapatellar plica (white arrow) appears as a curvilinear high signal passing through Hoffa's fat pad (not detected by ultrasound). P, patella; PT, patellar tendon

infrapatellar bursa. In the present study, the sensitivity and specificity of ultrasonography were $66.7 \%$ and $100 \%$, respectively.

Our results matched Blankstein et al. [38] in the assessment of the bipartite patella and reported $100 \%$ sensitivity and specificity of ultrasonography.

Gel stand-off pad is an aqueous, flexible, easily available, disposable spacer, widely used in B-mode ultrasonography approach of superficial lesions or difficult-tovisualize areas. Moreover, it allows the detection of otherwise-missed peri- or intra-lesional flow signals on Doppler imaging [39]. In our study, we did not use the gel pad and used plenty of thick gel to avoid loss of contact. Therefore, further studies discussing the value of using gel stand-off pad in musculoskeletal ultrasonography are recommended.
Although ultrasonography had high diagnostic accuracy, a substantial number of lesions were missed. The main lesions missed by ultrasonography and detected by MRI were the patellar cartilage defects (ultrasonography missed all lesions) and the trochlear cartilage defects (ultrasonography missed 9 lesions). Accordingly, ultrasonography can be used in the diagnosis and screening of patients with AKP and can be used as an alternative to MRI when MRI was unavailable or contraindicated. MRI is indicated if a patellar cartilage defect is clinically suspected or the ultrasonography yielded negative results.

Our study has some limitations: First, there was a wide variety of findings; some of these findings may not be the actual cause of the AKP, and the others had no previously published results in the literature 

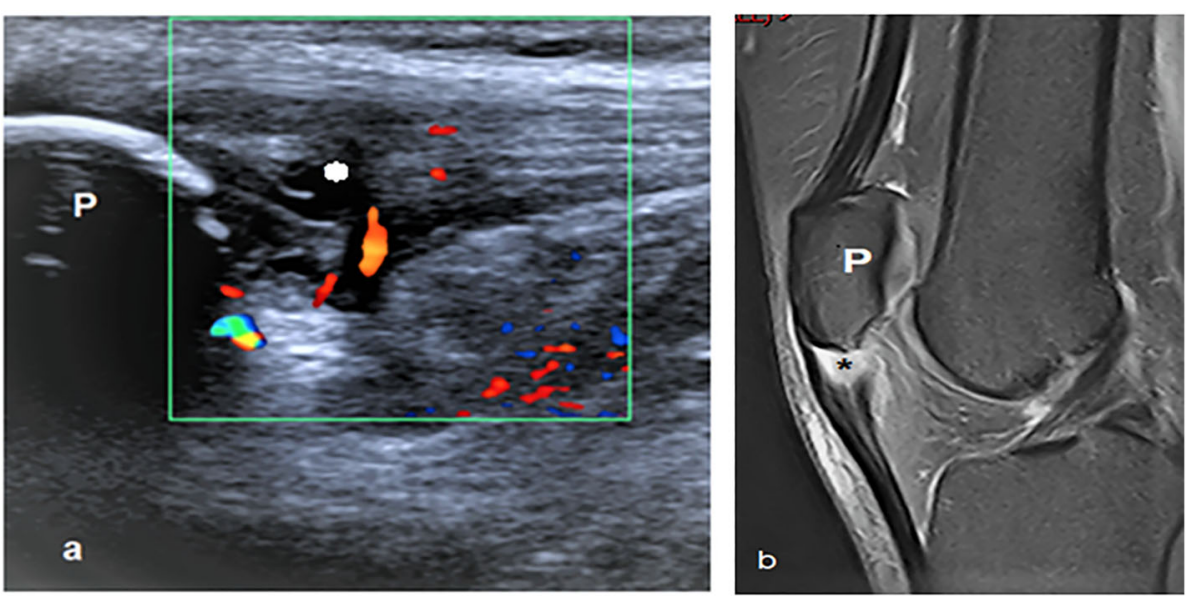

Fig. 9 A 22-year-old male with left patellar tendinopathy. a Long axis ultrasound image demonstrates the thickened proximal part of the patellar tendon with cystic changes $\left(^{*}\right)$. Color Doppler mapping shows increased vascularity in and around the tendon. b Sagittal PDFS shows thickened proximal patellar tendon with fluid signal $\left(^{*}\right)$. P, patella

to compare. This generalizability may result in substantial compromises on the quality of findings. However, this study was an attempt to publish a comprehensive review about the diagnostic accuracy of ultrasonography in patients with AKP. Second, there was higher sensitivity of ultrasonography in detecting joint effusion, which was at the same time the most common finding in the current study. The perfection of ultrasonography in detecting joint effusion increased the overall sensitivity of ultrasonography in the detection of pathological findings in the AKP patients and masked its weakness in detecting other entities like patellar cartilage defect and infrapatellar plica. Third, unfortunately, all the ultrasonography examinations were performed by one radiologist. Hence, there was no chance to make the intra- and interobserver agreement. Further studies discussing the possible variability of ultrasonographic signs among radiologists and providing data for operator intra- and inter-observer agreement are recommended. Fourth, the patients included in this study were already scheduled for an MRI examination, which could produce a selection bias. Nevertheless, not all patients scheduled for an MRI examination in this study were severed or complicated. Finally, lack of surgical data and clinical follow-up.

\section{Conclusion}

Ultrasonography can be used to diagnose patients with AKP; it showed high diagnostic accuracy in detecting
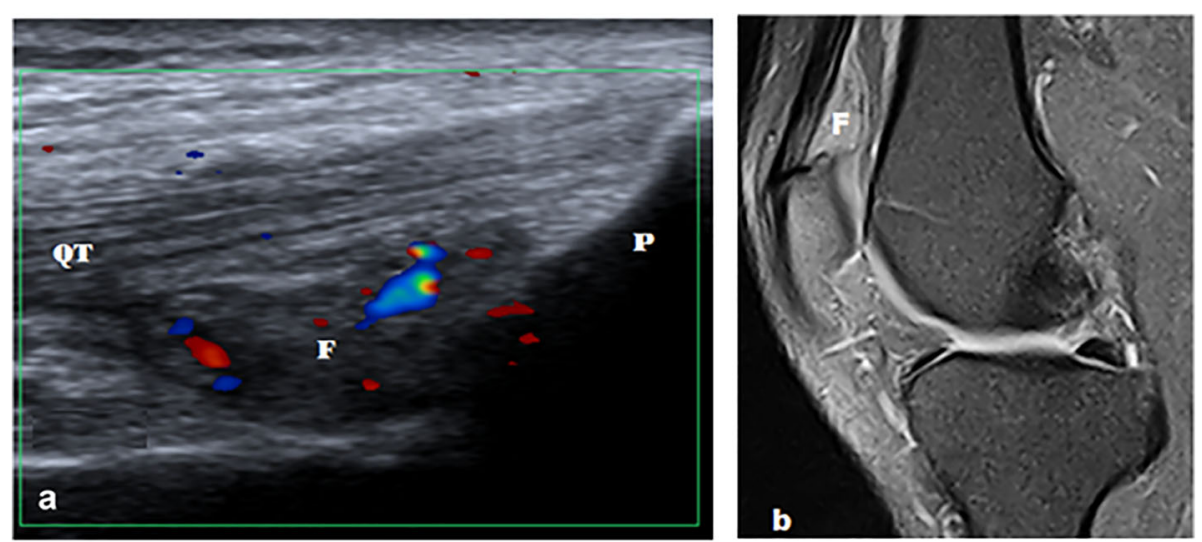

Fig. 10 A 37-year-old male with left suprapatellar pad of fat impingement. a Long axis ultrasound image shows hypoechoic suprapatellar fat pad with convex posterior margin. Color Doppler ultrasound shows increased vascularity. b Sagittal PDFS MR image shows hyperintense suprapatellar fat pad with convex posterior margin. QT, quadriceps tendon; P, patella; F, suprapatellar fat pad 
most of the findings. Although MRI is the gold standard technique for AKP imaging, ultrasonography can be used to make a swift screening and assessment of the painful anterior knee and can be used as an alternative to MRI when MRI is unavailable or contraindicated. MRI may be warranted if a patellar cartilage defect is clinically suspected or the ultrasonography yielded negative results.

\section{Abbreviations}

AKP: Anterior knee pain; AUC: Area under the curve; MRI: Magnetic resonance imaging; ROC: Receiver operating characteristic curve

\section{Acknowledgements}

The authors thank all staff members and colleagues in the Radiology Departments at Zagazig and Benha Universities for their helpful cooperation.

\section{Informed consent}

Written informed consent was obtained from all patients.

\section{Statistics and biometry}

The corresponding author has great statistical expertise.

\author{
Methodology \\ - Prospective. \\ - Diagnostic or prognostic study. \\ - Performed at a single center.
}

\section{Authors' contributions}

Guarantor of integrity of the entire study-(MAB). Study concepts and design-(MAB, DBE, SAA, HYA). Literature research-(TMA, NMM, SM). Clinical studies-(TMG, TA, SM, AME). Experimental studies/data analysis-(MAB, HYA, DBE, SAA, TMA, NMM, TMG, TA, SM, AME). Statistical analysis-(MAB, HYA). Manuscript preparation-(MAB, HYA). Manuscript editing - $(M A B, H Y A)$. All authors read and approved the final manuscript

\section{Funding}

The authors declare that this work has not received any funding.

\section{Availability of data and materials}

The datasets used and/or analyzed during the current study are available from the corresponding author on reasonable request.

\section{Ethics approval and consent to participate}

Institutional review board approval was obtained.

\section{Consent for publication}

Not applicable

\section{Competing interests}

The authors declare that they have no competing interests.

\section{Author details}

${ }^{1}$ Department of Radiodiagnosis, Faculty of Human Medicine, Zagazig University, Zagazig, Egypt. ${ }^{2}$ Department of Radiodiagnosis, Faculty of Human Medicine, Benha University, Benha, Egypt. ${ }^{3}$ Department of Orthopaedic Surgery, Faculty of Human Medicine, Ain Shams University, Cairo, Egypt. ${ }^{4}$ Department of Orthopaedic Surgery, Faculty of Human Medicine, Tanta University, Tanta, Egypt. ${ }^{5}$ Department of Rheumatology and Rehabilitation, Faculty of Human Medicine, Zagazig University, Zagazig, Egypt. ${ }^{6}$ Department of Psychiatry, Faculty of Human Medicine for Girls, Al-Azhar University, Zagazig, Egypt.
Received: 29 July 2020 Accepted: 16 September 2020

Published online: 01 October 2020

\section{References}

1. Sanchis-alfonso V (2014) Holistic approach to understanding anterior knee pain. Clinical implications. Knee Surg Sport Traumatol Arthrosc 22(10):22752285

2. Whitlock K, Mosier B, Matzkin E (2018) Anterior Knee Pain: Diagnosis and Treatment. In: Katz J., Blauwet C., Schoenfeld A. (eds) Principles of Orthopedic Practice for Primary Care Providers. Springer, Cham. 19:313-327

3. Samim M, Smitaman E, Lawrence D, Moukaddam H (2014) MRI of anterior knee pain. Skeletal Radiol 43(7):875-893

4. Sanchis-Alfonso V, Dye SF (2017) How to deal with anterior knee pain in the active young patient. Sports Health 9(4):346-351

5. Hosny S, McClatchie W, Sofat N, Hing BC (2012) Knee pain in adults \& adolescents, diagnosis and treatment. Pain Perspect. October. IntechOpen

6. Sanchis-Alfonso V, Ordoño F, Subías-López A, Monserrat C (2011) Pathogenesis of anterior knee pain in the active young: is there a relation between the presence of patellofemoral malalignment and pain? In: Sanchis-Alfonso V (eds) Anterior knee pain and patellar instability. Springer, London, pp 51-60

7. Sanchis-Alfonso V, McConnell J, Monllau JC, Fulkerson JP (2016) Diagnosis and treatment of anterior knee pain. Journal of ISAKOS: Joint Disorders \& Orthopaedic Sports Medicine 1(3):161-173

8. Ostlere S (2007) Imaging of anterior knee pain and maltracking. In: imaging of orthopedic sports injuries. In: Vanhoenacker FM, Maas M, Gielen JL (eds) Imaging of Orthopedic Sports Injuries. Medical Radiology (Diagnostic Imaging). Springer, Berlin, Heidelberg

9. De Smet E (2017) Imaging of anterior knee pain. J Belgian Soc Radiol 101:1-2

10. Terslev L, Qvistgaard E, Torp-Pedersen S, Laetgaard J, Danneskiold-Samsoe B, Bliddal H (2001) Ultrasound and power Doppler findings in jumper's knee - preliminary observations. Eur J Ultrasound 13(3):183-189

11. Fredberg $U$, Bolvig $L$ (2002) Significance of ultrasonographically detected asymptomatic tendinosis in the patellar and Achilles tendons of elite soccer players: a longitudinal study. Am J Sports Med 30(4):488-491

12. Hong A, Liu JN, Gowd AK, Dhawan A, Amin NH (2019) Reliability and accuracy of MRI in orthopedics: a survey of its use and perceived limitations. Clin Med Insights Arthritis Musculoskelet Disord 12: 1179544119872972

13. Escala JS, Mellado JM, Olona M, Giné J, Saurí A, Neyret P (2006) Objective patellar instability: MR-based quantitative assessment of potentially associated anatomical features. Knee Surg Sports Traumatol Arthrosc 14(3): 264-272

14. Corvino A, Catalano O, Corvino F, Sandomenico F, Setola SV, Petrillo A (2016) Superficial temporal artery pseudoaneurysm: what is the role of ultrasound? J Ultrasound 19(3):197-201

15. Corvino A, Corvino F, Catalano O, Sandomenico F, Petrillo A (2017) The tail and the string sign: new sonographic features of subcutaneous melanoma metastasis. Ultrasound Med Biol 43(1):370-374

16. Catalano O, Alfageme Roldán F, Varelli C, Bard R, Corvino A, Wortsman X (2019) Skin cancer: findings and role of high-resolution ultrasound. J Ultrasound 22(4):423-431

17. DeLong ER, DeLong DM, Clarke-Pearson DL (1988) Comparing the areas under two or more correlated receiver operating characteristic curves: a nonparametric approach. Biometrics 1:837-845

18. Altman DG (1991) Practical statistics for medical research. Chapman and $\mathrm{Hall} / \mathrm{CRC}$

19. Artul S, Khazin F, Hakim J, Habib G (2014) Ultrasonographic findings in a large series of patients with knee pain. J Clin Imaging Sci 4(1):45

20. Lee MJ, Chow K (2007) Ultrasound of the knee. Semin Musculoskelet Radiol 11(2):137-148

21. Draghi F, Ferrozzi G, Urciuoli L, Bortolotto C, Bianchi S (2016) Hoffa's fat pad abnormalities, knee pain and magnetic resonance imaging in daily practice. Insights Imaging 7(3):373-383

22. Cao J, Zheng B, Meng X et al (2018) A novel ultrasound scanning approach for evaluating femoral cartilage defects of the knee: comparison with routine magnetic resonance imaging. J Orthop Surg Res 13(1):1-12

23. Unlu EN, Turhan Y, Kos DM, Safak AA (2017) Assessment of anterior subcutaneous hypersignal on proton-density-weighted MR imaging of the knee and relationship with anterior knee pain. Diagn Interv Imaging 98(4): 339-345 
24. Lee P, Nixion A, Chandratreya A, Murray J (2017) Synovial plica syndrome of the knee: a commonly overlooked cause of anterior knee pain. Surg J (N Y) 3(01):e9-e16

25. Kheiralla OAM (2016) Imaging appearances of synovial plicae syndrome of the knee. Int J Med Res Heal Sci 5(8):140-149

26. Schwartz A, Watson JN, Hutchinson MR (2015) Patellar tendinopathy. Sports Health 7(5):415-420

27. Warden SJ, Kiss ZS, Malara FA, Ooi ABT, Cook JL, Crossley KM (2007) Comparative accuracy of magnetic resonance imaging and ultrasonography in confirming clinically diagnosed patellar tendinopathy. Am J Sports Med 35(3):427-436

28. Friedman L, Finlay K, Popovich T, Chhem RK (2003) Sonographic findings in patients with anterior knee pain. J Clin Ultrasound 31(2):85-97

29. King D, Yakubek G, Chughtai M et al (2019) Quadriceps tendinopathy: a review-part 1: epidemiology and diagnosis. Ann Transl Med 7(4):71-71

30. Jacobson JA, Lenchik L, Ruhoy MK, Schweitzer ME, Resnick D (1997) MR imaging of the infrapatellar fat pad of Hoffa. Radiographics 17(3):675-691

31. Tsavalas N, Karantanas AH (2013) Suprapatellar fat-pad mass effect: MRI findings and correlation with anterior knee pain. AJR Am J Roentgenol 200(3):W291-W296

32. Gallagher J, Tierney P, Murray P, O'Brien M (2005) The infrapatellar fat pad: anatomy and clinical correlations. Knee Surg Sports Traumatol Arthrosc 13(4):268-272

33. Gutierrez LB, Morgan TA, Link T, Feeley B, Motamedi D (2016) Utility of musculoskeletal ultrasound in the diagnosis and treatment of suprapatellar fat pad impingement: a case report. J Clin Case Rep. 6(837):2

34. Gutierrez LB, Morgan TA (2016) Utility of musculoskeletal ultrasound in the diagnosis and treatment of suprapatellar fat pad impingement: a case report. J Clin Case Reports 6(7):7-9

35. Tsavalas N, Karantanas AH (2013) Suprapatellar fat-pad mass effect: MRI findings and correlation with anterior knee pain. AJR Am J Roentgenol 200(3):291-296

36. Mikkilineni H, Delzell PB, Andrish J et al (2018) Ultrasound evaluation of infrapatellar fat pad impingement: an exploratory prospective study. Knee 25(2):279-285

37. Draghi F, Corti R, Urciuoli L, Alessandrino F, Rotondo A (2015) Knee bursitis: a sonographic evaluation. J Ultrasound 18(3):251-257

38. Blankstein A, Cohen I, Salai M, Diamant L, Chechick A, Ganel A (2001) Ultrasonography: an imaging modality enabling the diagnosis of bipartite patella. Knee Surg Sport Traumatol Arthrosc 9(4):221-224

39. Corvino A, Sandomenico F, Corvino F, Campanino MR, Verde F, Giurazza F, Catalano O (2020) Utility of gel stand-off pad in the detection of Doppler signal on focal nodular lesions of the skin. J Ultrasound 23(1):45-53

\section{Publisher's Note}

Springer Nature remains neutral with regard to jurisdictional claims in published maps and institutional affiliations.

\section{Submit your manuscript to a SpringerOpen ${ }^{\circ}$ journal and benefit from:}

- Convenient online submission

- Rigorous peer review

- Open access: articles freely available online

- High visibility within the field

- Retaining the copyright to your article

Submit your next manuscript at $\boldsymbol{\nabla}$ springeropen.com 